ARTÍCULO ORIGINAL

\title{
Incidencia de distintas frecuencias de corte en parámetros nutritivos de pasturas de alfalfa (Medicago sativa L.) de distinto grado de reposo invernal
}

\author{
Ordoñez $\mathrm{VN}^{1^{*}}$, Nescier $\mathrm{IM}^{2}$, Dovis $\mathrm{VL}^{2}$, Gieco $\mathrm{JO}^{1}$, Bonvin $\mathrm{C}^{1}$, Rainaudo $\mathrm{MB}^{1}$ \\ ${ }^{1}$ Facultad de Ciencias Agrarias, Universidad Nacional del Litoral (FCA-UNL), Argentina. \\ 2 Centro APTA Citros "Sylvio Moreira", Instituto Agronômico, Cordeirópolis, SP, Brasil. \\ * Correspondencia: Vanesa Ordoñez. FCA-UNL, RP Kreder 2805, 3080 Esperanza, Santa Fe, Argentina. \\ E-mail: vanesaord@fca.unl.edu.ar
}

Recibido: 14 Mayo 2020. Aceptado: 12 de Diciembre 2020. Disponible en línea: 1 Marzo 2021

Editor: P. Beldomenico

RESUMEN. El objetivo de este trabajo fue evaluar la calidad nutricional en 3 cultivares de alfalfa, pertenecientes a diferentes grupos de reposo invernal (GRI), sometidos a dos frecuencias de corte, durante un año del cultivo, fundamentado en que es abundante la información existente en estudios realizados en productividad, cobertura y persistencia, no así en la determinación de calidad de forraje sometidos a diferentes frecuencias de cortes y de GRI contrastantes. Se utilizaron tres cultivares (GR6-Verzy), (GR9-Mecha) y (GR10-Ruano). El diseño experimental fue de parcelas divididas con cuatro repeticiones. Se establecieron 2 tratamientos definidos por los intervalos entre cortes: T1: 25 días y T2: 35 días. Estos fueron precisados para las estaciones de primavera, verano y otoño. En invierno el intervalo fue de 45 y 55 días en T1 y T2, respectivamente. Las variables evaluadas fueron: porcentaje de materia seca (\% MS), porcentaje de proteína bruta (\% PB), porcentaje de fibra detergente neutro (\% FDN) y porcentaje de fibra detergente ácido (\% FDA). Se encontraron diferencias significativas para todas las variables nutricionales estudiadas, para ambos tratamientos. Se observó interacción entre cultivares y tratamiento para \% PB. Los cultivares estudiados con diferentes GRI, no expresaron diferencias significativas en los parámetros de calidad evaluados para ninguna de las frecuencias de corte realizadas. La frecuencia de corte de 35 días arrojó los menores \% PB y mayores \% FDN y \% FDA, por lo cual el forraje resulta para esta frecuencia de menor calidad, llevando a la disminución de la digestibilidad y aprovechamiento de la pastura para el ganado.

SUMMARY. Incidence of cutoff frequency in nutritional parameters of alfalfa (Medicago sativa L.) of different degrees of winter rest. The objective of this work was to evaluate the nutritional quality in 3 cultivars of alfalfa, belonging to different winter dormancy (GRI), subjected to two cutting frequencies during one year of cultivation, based on the abundant information existing in studies carried out in productivity, coverage and persistence, not so in the determination of quality of forage subjected to different frequencies of cuts and contrasting GRI. Three cultivars (GR6-Verzy), (GR9-Mecha) and (GR10-Ruano) were tested. The experimental design was a complete split plot with four repetitions. Two treatments defined by the cutting intervals were established: T1: 25 days and T2: 35 days. These were specified for spring, summer and fall seasons. For winter the interval was 45 and 55 days in T1 and T2, respectively. The evaluated variables were: percentage of dry matter (\% DM), percentage of crude protein (\% PB), percentage of neutral detergent fiber (\% FDN) and percentage of acid detergent fiber (\% FDA). Significant differences were found for all the nutritional variables studied, for both treatment with $p<0.05$ with InfoStat program. Interaction between cultivars and treatment was observed for \% PB. The 35 days cutoff frequency of yielded the lowest percentages of PB and the highest percentages of FDN and FDA. Hence the forage resulting from this frequency is of a lower quality, leading to decreased digestibility and lower performance of pasture for livestock.

Palabras clave: alfalfa, cultivar, valor nutritivo, frecuencia de corte

Keywords: alfalfa, cultivate, nutritional parameters, cutting frequency

\section{Introducción}

Los sistemas de producción ganadera de Argentina están basados en el pastoreo directo de los recursos forrajeros, con ocasional uso de suplementos tales como granos, subproductos de cosecha y forrajes conservados como heno o silaje. En la Cuenca Lechera Santafesina la alimentación del ganado bovino se basa fundamentalmente en la utilización de forrajes verdes, como alternativa de bajo costo en relación a otros alimentos; siendo la alfalfa (Medicago sativa L.) el recurso forrajero más utilizado (Quiñoñez et al., 2003).

En el caso de las pasturas y los forrajes conservados, variaciones en la calidad se registran debido a la especie, época del año, estado fisiológico de las plantas, el momento de corte o de pastoreo, así como tipo y cantidad de fertilizante aplicado. El estudio del efecto de estos factores sobre la calidad de los forrajes es de vital importancia para caracterizar nutricionalmente los 
mismos, de tal manera que se optimice la producción (Colombatto, 2020).

Una de las características más relevantes de la alfalfa como especie forrajera es su elevado aporte de proteínas, además de vitaminas y minerales, lo que determina alto valor nutricional y digestibilidad (Mccoy y Walker, 1984). Entre los factores que afectan esta calidad se encuentran la relación hoja/tallo y la concentración de fibras y lignina; siendo que ambos se modifican con el grado de madurez fisiológica. Con el avance de la edad fisiológica ocurre una reducción de la relación hoja/tallo y aumento de la concentración de fibras y lignina, principales componentes de las estructuras del tallo, a la vez que hay una disminución en \% PB (INIA La Estanzuela, 2000 y Mieres et al., 2000).

Actualmente, con sistemas de producción más intensivos y exigentes, resulta necesario obtener pasturas de alta calidad y productividad a fin de satisfacer las necesidades del ganado de alta performance productiva. En este sentido, cobran relevancia estudios que permitan ajustar los momentos de pastoreo o de corte de las pasturas, con el objetivo de maximizar la calidad y realizar un eficiente uso de las mismas (Nelson y Moser, 1994). Romero et al. (1995) sostienen que para obtener una alta cantidad y calidad de forraje, debe usarse una frecuencia de defoliación que permita a la planta producir y al mismo tiempo recuperarse, entre cortes o pastoreos. Cuando se aumenta el intervalo entre cortes se aumenta la producción de forraje en detrimento de la calidad y digestibilidad, frecuencias de corte mayores conducen al agotamiento de las plantas y reducción de la vida útil (Ball et al., 2001). Chen et al. (2012) encontraron con intervalos de corte de 40 días los mejores resultados en rendimiento y calidad en cultivares de alfalfa del mismo GRI, comparado con frecuencias de 30 y 60 días. Al mismo tiempo recomiendan la importancia de encontrar prácticas óptimas de manejo que aumenten el rendimiento y lograr el equilibrio entre mayor calidad y productividad.

Para la evaluación de la calidad se han desarrollado métodos de análisis basados en las propiedades físicas y químicas de los forrajes. Un grupo importante de técnicas de análisis de calidad forrajera está basado en la determinación de la cantidad relativa de pared y de contenidos celulares. Para los rumiantes, la celulosa y la hemicelulosa son parcialmente digestibles, en tanto que la lignina es indigestible. Por otro lado, los contenidos celulares (citoplasma) son la parte de mayor valor nutritivo y de más alta digestibilidad, proveyendo el mayor \% PB, carbohidratos solubles y lípidos (Basigalup et al., 2007)

El método de fibra detergente neutro y fibra detergente ácido desarrollado por Goering y Van Soest (1970), es considerado un patrón para la caracterización de la calidad de forrajes. La FDA representa la fracción menos digestible del alimento y se relaciona inversamente con la digestibilidad, mientras que la FDN al comprender toda la pared celular puede ser considerada como predictora del consumo al incluir a la fracción menos digestible y a la lentamente digestible (Fahey y Berger, 1988 y Van Soest, 1994). Aufrère et al. (2000) y Cassida et al. (2000) destacan que el contenido de FDN y de FDA para el forraje de alfalfa se incrementa con la madurez de la planta mientras el contenido de $\mathrm{N}$ y la digestibilidad de la materia orgánica decrecen en los cortes de estado vegetativo y floración, hallando valores intermedios en el rebrote siguiente.

Las variedades de alfalfa disponibles en el mercado tienen diferencias en el grado de reposo invernal o latencia, lo que determina diferencias en la distribución estacional del forraje y en la calidad. De esta manera se hace necesario establecer un sistema de manejo para cada grado de latencia varietal, siendo que el uso de variedades con ciclos de crecimiento contrastantes hace más complejo y especializado al mismo (INIA La Estanzuela, 2000). Ventroni et al. (2010) sostienen que la productividad y la superviviencia varían según la frecuencia de corte, aunque está poco estudiada la incidencia de la frecuencia en el rendimiento del forraje y componentes de rendimiento. Así es necesario un profundo conocimiento de las respuestas al ambiente de variedades de diferentes grupos de latencia a fin de establecer las mejores alternativas de manejo que permitan producir la mayor cantidad y calidad de forraje. Al respecto Ordoñez et al., (2019) evaluaron la incidencia de diferentes frecuencias de corte en la productividad, cobertura y persistencia en 3 variedades de alfalfa, encontrando que a frecuencias de corte mayores aumenta la productividad en detrimento de la persistencia del forraje. Son escasos los estudios que evalúen calidad en cultivares con diferentes GRI sometidos a diferentes frecuencias de corte. Chen et al. (2012) evaluó calidad de pastura, con diferentes frecuencias de corte comparando cultivares de un mismo GRI. Ventroni et al. (2010) evaluaron productividad y persistencia a diferentes frecuencias de corte con distintos GRI en la localidad de Balcarce, pero no evaluaron parámetros de calidad.

El objetivo de este trabajo fue evaluar la variación estacional en la calidad nutricional de 3 cultivares de alfalfa de diferentes grupos de reposo invernal, utilizadas en la Cuenca Lechera Santafesina sometidos a dos frecuencias de corte.

\section{Materiales y métodos}

\section{Caracterización del sitio}

La pastura se implantó en el campo experimental de Producción Animal de la Facultad de Ciencias Agrarias de la Universidad Nacional del Litoral, ubicado en la localidad "Rincón de Ávila", sobre ruta Provincial № 6 $(\mathrm{km} \mathrm{81,5),} \mathrm{a} \mathrm{31ㅇ} \mathrm{20'} \mathrm{08"} \mathrm{latitud} \mathrm{Sur,} \mathrm{60ㅇ} \mathrm{50'} \mathrm{48"}$ longitud Oeste y a una altitud de $34 \mathrm{~m}$ s.n.m., en la Región Centro Norte de la Provincia de Santa Fe, Argentina. La siembra se realizó en el mes de agosto del año 
2017 sobre un suelo Argiudol Típico (Panigatti, 2010) y el período de cortes se extendió desde el mes de septiembre de 2018 a junio de 2019. Los parámetros de calidad del suelo mostraron valores de $\mathrm{pH}=5,88 ; \mathrm{MO}$ $3,61 \%$; $N$ total $0,18 \%$; S 12,3 ppm SO $_{4}^{-2}$ y P 31,4 ppm disponible.

\section{Caracterización climática}

El clima de la zona presenta una temperatura media anual de $18 \stackrel{\circ}{\circ} \mathrm{C}$, donde la media del mes más cálido es 25 ㄷ y la del mes más frío 7 oC y la precipitación media anual es $1070 \mathrm{~mm}$ concentrados en el semestre que abarca desde el mes de octubre a marzo, donde se registra el $69 \%$ del total acumulado anualmente. Los meses estivales (febrero-marzo) presentan los mayores registros, mientras que el trimestre invernal (junio-julioagosto) acumula los menores registros. Los datos para el período de ensayo se muestran en la Figura 1 (Estación meteorológica FCA, Pegasus).

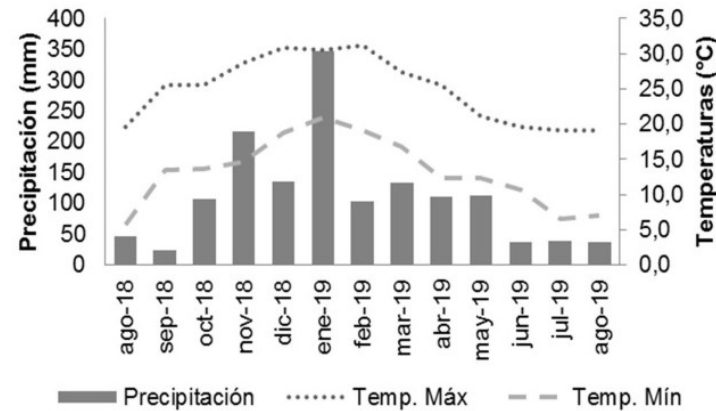

Figura 1. Registro de medias de temperatura y precipitaciones mensuales durante el período de ensayo.

\section{Establecimiento del cultivo, diseño experimental y tratamientos}

Dada las condiciones de fertilidad del suelo, la práctica de fertilización no se consideró necesaria. Previo a la siembra se realizaron trabajos de preparación del suelo con la finalidad de lograr una correcta germinación, emergencia y alto porcentaje de establecimiento. Se utilizó una densidad de siembra de $14 \mathrm{~kg} / \mathrm{ha}$ de semilla peleteada conteniendo inoculante (Rhizobium meliloti), fungicida, insecticida y cubierta de carbonato de calcio. El espaciamiento entre hileras fue de $17 \mathrm{~cm}$.

Se utilizaron tres cultivares comerciales de alfalfa, uno de reposo invernal intermedio (GR6-Verzy) y dos sin reposo invernal (GR9-Mecha) y (GR10-Ruano), en esta elección se buscó comparar diferencias entre los distintos GRI, que son de uso frecuente en la región. EI diseño experimental fue de parcelas divididas con arreglo en bloques completos, cuatro repeticiones. Las parcelas son los cultivares de alfalfa utilizados, y las subparcelas estuvieron determinadas por las frecuencias de corte aplicadas. El tamaño de cada parcela evaluada (variedad-frecuencia de corte) fue de $2 \mathrm{~m}$ de ancho por $5 \mathrm{~m}$ de largo.

\section{Características de las especies estudiadas}

GRI 6-Verzy:

- $\quad$ Reposo invernal intermedio.

- Superior estabilidad productiva en distintos ambientes. Ideal para planteos combinados de pastoreo con reservas forrajeras.

- Resistente a enfermedades foliares y bacteriosis.

GRI 9-Mecha:

- $\quad$ Sin reposo invernal.

- Excelente potencial productivo en sistemas intensivos de pastoreo.

- Resistente a enfermedades foliares y bacteriosis.

GRI 10-Ruano:

- Extremadamente sin reposo invernal.

- Excelente adaptación productivo en sistemas intensivos de pastoreo.

- Resistente a enfermedades foliares y bacteriosis.

\section{Tratamiento y manejo}

Se establecieron 2 tratamientos definidos por los intervalos de corte: T1: 25 días y T2: 35 días, que fueron establecidos teniendo en cuenta las frecuencias utilizadas en la zona, partiendo el ensayo con un corte inicial (Inic), común a ambos tratamientos. Estos fueron establecidos para las estaciones de primavera, verano y otoño. En invierno, debido a la menor tasa de acumulación de biomasa de la planta se utilizó un intervalo de corte de 45 y 55 días necesarios para la recuperación de la pastura en $\mathrm{T} 1$ y $\mathrm{T} 2$, respectivamente. La fecha del último corte, antes del descanso, para T1 fue el 4 de mayo, y para T2 el 23 de abril. En el período de ensayo se realizaron 11 cortes para T1 y 8 cortes para T2. Estableciendo un corte por estación para evaluar calidad. La altura de corte fue de $8 \mathrm{~cm}$.

\section{Análisis químicos}

Los parámetros evaluados fueron:

Porcentaje de materia seca (\% MS): Se realizó sobre muestras compuestas de $200 \mathrm{~g}$ por parcela, que fueron secadas en estufa a 55ㄷ C hasta peso constante según Promefa (Jaurena y Wawrzkiewicz, 2009).

Concentración de fibras: Las determinaciones de \% FDN y \% FDA se realizaron siguiendo el protocolo de Van Soest y Wine, R. H. (1967).

Proteína bruta: El contenido de Proteína bruta (\% PB = $\% \mathrm{~N}$ Kjeldahl $\times 6,25)$ se realizó mediante digestión con ácido sulfúrico concentrado (AOAC, 1990). 


\section{Tratamiento estadístico}

Los resultados se analizaron con uso del programa Infostat (Di Rienzo et al., 2016) por medio de análisis de Varianza factorial con interacción (ANAVA) con dos fuentes de variación (frecuencias de corte y cultivares), cuando el modelo detectó diferencias significativas, estas fueron sometidas a Test de Tukey.

\section{Resultados y Discusión}

Se observaron diferencias significativas entre los tratamientos para todas las variables nutricionales estudiadas con $\mathrm{p}<0,05$ (Fig.2), y sin diferencias entre los promedios anuales de las distintas variedades dentro de cada tratamiento. Con la frecuencia de corte de 35 días la calidad del forraje producido fue menor, con menor \% PB y mayor \% FDN y \% FDA, lo que lleva a la disminución en la digestibilidad y el aprovechamiento de la masa seca por el ganado. La reducción en la calidad y el aumento en la producción de forraje con frecuencias de corte mayores es conocida por los productores (Ball et al., 2001). Sin embargo, a pesar de la reducción en la calidad los \% FDA observados en T2 fueron menores a los valores medios destacados en la Tabla de Composición Química de Alimentos para Rumiantes EEA Rafaela (2008). Por otro lado, cuando son usadas frecuencias mayores la cantidad de fibras es menor y así mejora la calidad en detrimento de la persistencia de la pastura, según Ordoñez et al., 2019.

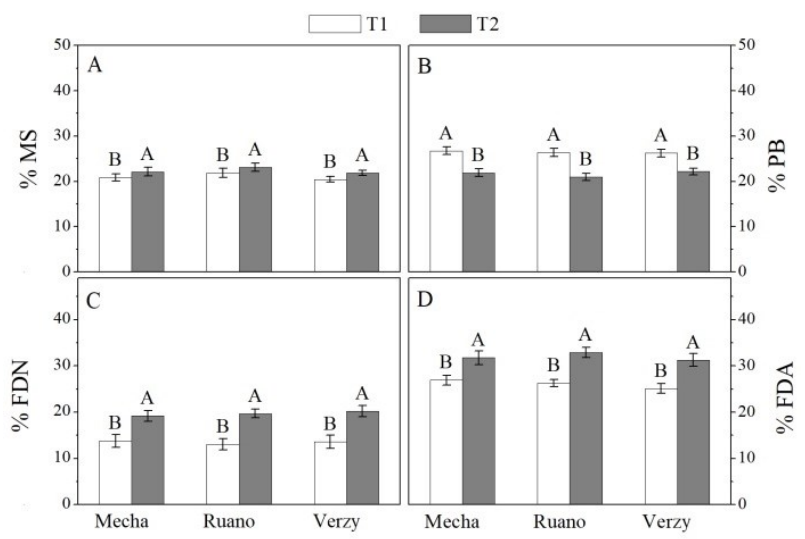

Figura 2. Porcentaje promedio de: A- materia seca (\%MS), Bproteína bruta (\%PB), C- fibra detergente neutra (\%FDN) y Dfibra detergente ácida (\%FDA) durante el período de ensayo en cultivares de alfalfa de diferente grado de reposo invernal, sometidos a dos frecuencias de corte: 25 días entre cortes (T25) y 35 días entre cortes (T35). Letras distintas indican diferencias significativas para cada cultivar entre los tratamientos (Test de Tukey, $\mathrm{p}<0,05$ ).

Como puede observarse en la figura 2, las tres variedades con GRI diferentes, tuvieron comportamientos similares para las distintas frecuencias de corte a lo largo del año.
Analizado el comportamiento de las variables y considerando los valores obtenidos en cada uno de los momentos de corte se observaron diferencias entre las fechas de corte y tratamientos de frecuencia de corte en análisis de variancia con interacción de los factores $(p<0,05)$, siendo los coeficientes de variación máximos y mínimos obtenidos de $17,2 \%$ y $6.1 \%$. Al mismo tiempo hubo interacción entre estos dos factores para las variables \% PB y FDN $(p<0.05)$, por este motivo se procedió a realizar nuevos ANOVAs independientes para cada frecuencia de corte.

Cuando se analizó el comportamiento estacional, de las variables estudiadas en los tres cultivares utilizados, se pudo comprobar que hubo diferencias entre las medias en las características nutritivas a lo largo del año de forma similar para las tres variedades estudiadas con $p<0,1$ (Fig. 3). Los tres cultivares tuvieron pérdida de calidad nutritiva en verano como consecuencia de la reducción en la concentración del \% PB y aumento en la concentración de fibras (Fig 3). Mieres et al. (2000), expresan que para un buen comportamiento animal podría afirmarse que los valores proteicos son suficientes aun cuando se produce una caída en los valores nutritivos de la pastura, según el momento de corte de alfalfa. Los mayores \% PB se obtuvieron en los cortes de otoño para el cultivar (GRI 6) Verzy y el menor para el cultivar (GRI 10) Ruano, con contenidos del 24,0 \% y 23,2 \%, respectivamente, expresando la misma tendencia en los otros cortes. Para Rossanigo et al. (1995) la distribución de forraje es diferente entre grupos de reposo, siendo que los materiales sin reposo presentan mayor crecimiento en otoño e invierno que los grupos con reposo. Estos a su vez experimentan mayor acumulación de biomasa durante primavera y verano.

El grado de reposo invernal o latencia indica el periodo en el que la alfalfa no produce, ya que las variedades de diferentes grupos inician y finalizan el reposo con distintos umbrales de temperatura y longitud del día en el período de otoño/invierno (INIA La Estanzuela, 2000). Al respecto, durante el período del ensayo no se registraron temperaturas extremas persistentes para las estaciones de invierno y verano. Esta situación climática podría haber incidido en el comportamiento de los cultivares estudiados, con diferentes GRI, en la zona del centro-norte Santafesino, no expresando diferencias en los restantes parámetros de calidad.

En la Figura 3 puede observarse que al evaluar los parámetros nutritivos, entre cortes, en ambas frecuencias $(p<0,1)$, la calidad del forraje se deteriora mucho en verano y la mejor calidad se logra en primavera, coincidentemente con Montoya Buenrostro (2011) que concluye que la calidad de la pastura de alfalfa es muy variable y depende principalmente de la época del año, debido a que en los meses más cálidos la madurez se alcanza más rápidamente y la calidad del forraje desciende apresuradamente. 
$\mathrm{T} 1$
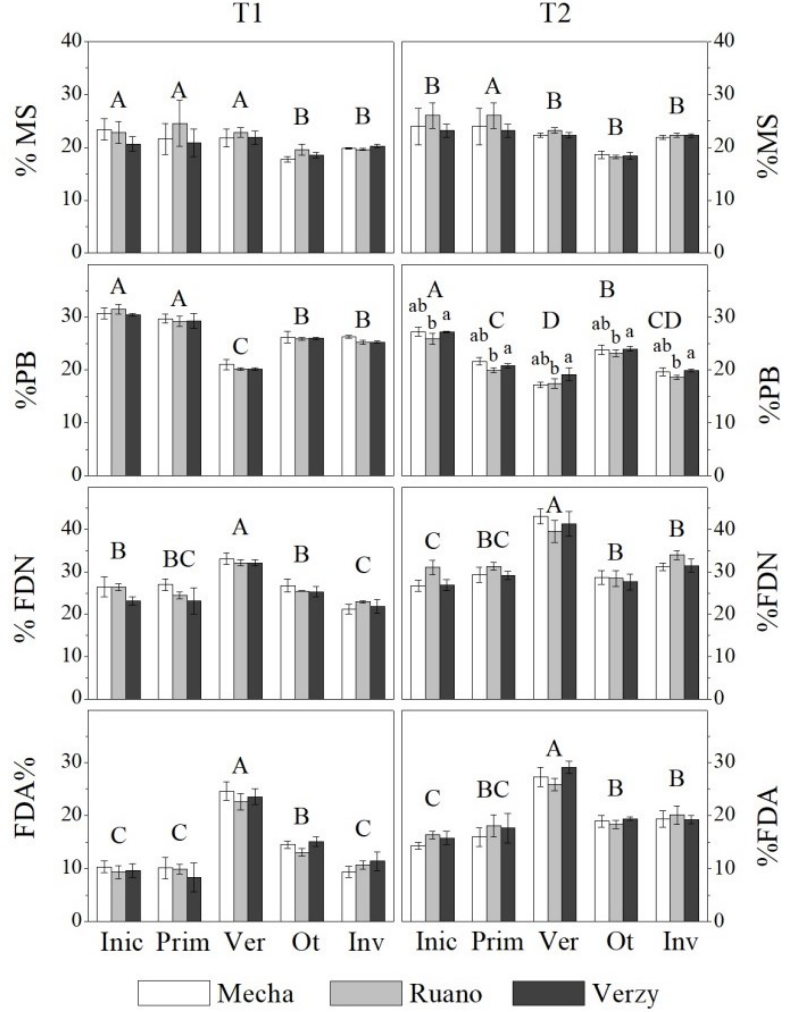

Figura 3. Medias de \%MS, \%PB, \% FDN y \% FDA para los cultivares utilizados en las diferentes fechas de cortes. Letras mayúsculas distintas indican diferencias significativas entre fechas de cortes para una misma variedad y letras minúsculas distintas indican diferencias significativas entre cultivares para una misma fecha de corte (Test de Tukey, $\mathrm{p}<0,1$ ).

Los cultivares estudiados con diferentes GRI, no expresaron diferencias significativas en los parámetros de calidad evaluados para ninguna de las frecuencias de corte realizadas, manifestándose solo diferencia en el contenido de PB para T2.

La pastura de alfalfa resultó de menor calidad en todos los cortes para T2. El cultivar Verzy para T2 mostró los mayores \% PB en todos los cortes, esta frecuencia probablemente permitió un mejor acúmulo de reservas y cobertura de plantas. Resultaría interesante investigar la asimilación de nitrógeno de este cultivar, a frecuencias de 35 días o mayores, que se ve reflejada en el aumento del contenido proteico.

Los mejores valores en los parámetros de calidad se obtuvieron para la estación de primavera.

\section{Bibliografía}

AOAC. 1990. Official Methods of analysis of the AOAC, 14th ed. AOAC Intl., Washington, DC. 2200 pp.

Aufrère J, Graviou D, Baumont R, Detour A, Demarquilly C. 2000. Degradation in the rumen of proteins from fresh lucerne forage in various stages of growth and conserved as silage or hay. Ann. Zootech. 49: 461-474.
Ball D, Collins M, Lacefield G, Martin N, Mertens D, Olson K, Putman D, Undersander D, Wolf M. 2001. Understanding Forage Quality. American Farm Bureau Federation Publication 1-01, Park Ridge, IL.

Basigalup DH, Rossanigo R, Ballario MV. 2007. Panorama actual de la alfalfa en la Argentina. En: Basigalup D H (Ed.). El cultivo de alfalfa en la Argentina. EEA Manfredi-INTA. Pp. 3-25.

Cassida KA, Griffin TS, Rodríguez J, Patching SC, Hesterman OB, Rust SR. 2000. Protein degradability and forage quality in maturing alfalfa, red clover, and birdsfoot trefoil. Crop Science 40: 209-215.

Chen J, Tang F, Zhu R, Gao C, Di G, Zhang Y. 2012. Effects of cutting frequency on alfalfa yield and yield components in Songnen Plain, Northeast China. African J. Biotechnol. 11: 4782-4790.

Colombatto D. 2020. Análisis de alimentos: Aplicaciones prácticas Departamento de Producción Animal, Facultad de Agronomía, Universidad de Buenos Aires. Av. San Martín 4453, C1417DSQ Buenos Aires, Argentina. (Material de enseñanza universitaria disponible en:

https://www.agro.uba.ar/sites/default/files/catedras/resumen colombatto.pdf 09/03/2020.)

Di Rienzo JA, Casanoves F, Balzarini MG, Gonzalez L, Tablada M, Robledo C W. 2016. InfoStat versión 2016. Grupo InfoStat, FCA, Universidad Nacional de Córdoba, Argentina. (Software informático disponible en: https://www.infostat.com.ar/)

Fahey GC, Berger LL. 1988. Carbohydrate nutrition in ruminants. En DC Church (Ed.). The ruminant animal, digestive physiology and nutrition. Prentice-Hall, Englewood Cliffs. Pp:69-297.

Goering HK, Van Soest PJ. 1970. Forage Fiber Analysis: Apparatus, Reagents, Pocedures and some Applications. USDAARS Agricultural Handbook 379, Washington DC. 24 pp.

Jaurena G, Wawrzkiewicz M. 2009. Guía de procedimientos analíticos. Programa para el mejoramiento de la evaluación de forrajes y alimentos - PROMEFA Centro de Investigación y Servicios en Nutrición Animal (CISNA) Departamento de Producción Animal Facultad de Agronomía - Universidad de Buenos Aires.

INIA La Estanzuela. 2000. Boletín de Divulgación N5 69. ${ }^{\circledR}$, INIA ISBN: 9974 -38 -109 -6 Editado por la Unidad de Difusión e Información Tecnológica del INIA. Montevideo- Uruguay.

INTA EEA Rafaela 2008. Tabla de Composición Química de Alimentos para Rumiantes. Gallardo, M, Romero, LA, Comerón, E. Compilado por Gaggiotti, M del C. CDD 636.086. Ediciones Instituto Nacional de Tecnología Agropecuaria. ISBN 978-987521-305-0.

Mccoy TJ, Walker ET. 1984 Cytology and cytogenetics of alfalfa. En: Hanson AA, Barnes DK and Hill R (Eds.). Alfalfa and alfalfa improvement. Agronomy Monographs 29, Madison, USA. Pp. 737-776.

Mieres JM, Rebuffo M, Risso DF, Restaino E. 2000. (Eds) Tecnología en allfalfa. CALIDAD DE FORRAJE. p.145-152. Programa Plantas Forrajeras, INIA La Estanzuela.. Boletín de Divulgación N 69. ${ }^{\circledR}$ 2000, INIA ISBN: 9974 -38 -109 -6 Editado 
por la Unidad de Difusión e Información Tecnológica del INIA. Andes 1365, Piso 12. Montevideo- Uruguay.

Montoya Buenrostro E. 2011. Variación en el contenido de nutrientes en alfalfa (Medicago sativa) cosechada a diferente edad de rebrote y época del año. (Tesis de maestría). Universidad Autónoma de Baja California, Instituto de Ciencias Agrícolas.

Nelson CJ, Moser LE. 1994. Plant factors affecting forage quality in Forage Quality, Evaluation, and Utilization. Based on the National Conference on Forage Quality, Evaluation, and Utilization held at the University of Nebraska, Lincoln, on 13-15 April 1994. Chapter 3: 115-154.

Ordoñez VN, Nescier I de los M, Dovis VL, Gieco JO, Bonvin C, Ribero G G, Walker G. 2019. Productividad y cobertura de cultivares de alfalfa (Medicago sativa L.) de diferentes grados de reposo invernal bajo dos frecuencias de corte. FAVE Sección Ciencias Agrarias 18: 8-15.

Panigatti J. 2010. 200 años, 200 suelos. Buenos Aires: INTA.

Quiñoñez AG, Dalla Fontana LA, Mollo, AJ. 2003. Respuesta de la alfalfa al agregado de fósforo, boro y calcio. FAVE Sección Ciencias Agrarias 2: 47-54.

Romero NA, Comeron E, Ustarroz E. 1995. Crecimiento y utilización de la alfalfa. En: Hijano E and Navarro A (Eds.). La Alfalfa en la Argentina. INTA C. R. Cuyo. Ed. Editar, San Juan. Pp. 151-170.

Rossanigo R, Spada M, Bruno O. 1995. Evaluación de cultivares de alfalfa y panorama varietal en la Argentina. En: Hijano E y Navarro A. Cap. 4. La alfalfa en la Argentina. Buenos Aires: INTA C.R. Cuyo. Pp 63-78.

Van Soest JP, Wine RH. 1967. Use of detergents in analysis of fibrous feeds IV. Determination of plant cell wall constituents. J. of the A.O.A.C. 50:55.

Van Soest JP. 1994. Nutritional ecology of the ruminant. 20 Ed. Cornell University Press, New York. 476 pp.

Ventroni LM, Jeffrey JV, Volenec JJ, Cangiano CA. 2010. Fall dormancy and cutting frequency impact on alfalfa yield and yield components. Field Crop Res. 119: 252-259. 